\title{
Inclusão digital de mulheres idosas longevas: uma experiência de empoderamento por meio da empatia na produção de tecnologia
}

\author{
Valéria Argôlo Rosa ${ }^{1}$, Luiz Felipe Rosário ${ }^{1}$, Igor Andrade ${ }^{1}$, Ecivaldo de Souza Matos ${ }^{1}$ \\ ${ }^{1}$ SPIDeLab/Departamento de Ciência da Computação - \\ Universidade Federal da Bahia (UFBA) \\ Salvador - BA - Brazil \\ \{valeria.rosa,ecivaldo\}@ufba.br, \{lfrosario,igorandrade83\}@gmail.com
}

\begin{abstract}
This paper presents an experience report of digital inclusion through an interaction codesign process for production of a mobile application. Ten elderly women, mostly long-lived (80+), with little or no experience in using digital technologies, participated in this experience. Participation in the interaction codesign process enables feelings of satisfaction, empowerment, and increased self-esteem, arousing the interest in being digitally included. We hope to contribute to other initiatives and studies on socio-digital inclusion.
\end{abstract}

Resumo. Este artigo apresenta um relato de experiência de inclusão digital por meio de um processo de codesign de interação para produção de um aplicativo móvel. Dez mulheres idosas participaram desta experiência, em sua maioria longevas (80+), com pouca ou nenhuma experiência no uso de tecnologias digitais. A participação no processo de codesign de interação possibilitou às participantes sentimentos de satisfação, empoderamento e aumento de autoestima, despertando o interesse em estarem incluídas digitalmente. Espera-se com este relato contribuir com outras iniciativas e estudos no campo da inclusão sociodigital.

\section{Introdução}

A população idosa vem crescendo aceleradamente. Estima-se que até o ano de 2060, 58,2 milhões de brasileiros, o equivalente a $25,5 \%$ da população brasileira, estarão com 65 anos ou mais [IBGE 2018]. Isso traz uma discussão em nível mundial sobre o que fazer para auxiliar pessoas idosas a se manterem saudáveis e ativas, especialmente nos países em desenvolvimento.

Segundo a Organização Mundial da Saúde (OMS) [Organization et al. 2005], uma pessoa é classificada como idosa a partir de 60 anos de idade, se residente em países em desenvolvimento; e a partir de 65 anos, se residente em países desenvolvidos.

Para esse período da vida, as tecnologias computacionais podem contribuir para o envelhecimento saudável e ativo. No entanto, [Pasqualotti et al. 2007] afirmam que pessoas idosas apresentam dificuldade em lidar com os avanços das tecnologias digitais e suas novas formas de linguagem. Mesmo equipamentos, como os smartphones e seus aplicativos, ainda são projetados para uso por pessoas jovens, ou seja, não consideram necessariamente o uso por pessoas idosas [Mol 2011]. 
Incluir digitalmente pessoas idosas é uma necessidade que tem se configurado como uma forma de socialização com o mundo contemporâneo, o que pode favorecer as relações familiares, sociais e comerciais; além de repercutir também na qualidade de vida do sujeito idoso, auxiliando principalmente nos seus estímulos cognitivos e motores [Bizelli et al. 2009].

Apesar do gigantesco número de telefones inteligentes circulando no país, uma parcela considerável dos brasileiros ainda os utilizam muito pouco, entre eles estão os idosos com mais de 55 anos que representa 17,21\% da população brasileira (IBGE, 2016). No entanto, verifica-se um crescimento desse grupo de população na utilização de recursos tecnológicos, como os smartphones. Dado o número crescente de idosos utilizando novas tecnologias, é fundamental gerar sistemas que contemplem as suas necessidades e expectativas [Lindsay et al. 2012].

[Simonsen and Robertson 2012] nos mostram que o Design Participativo (DP) pode ser uma solução viável para desenvolvimento de novas tecnologias, pois visa incluir potenciais usuários e outras partes interessadas durante todas as fases de design. Entretanto, [Muller and Druin 2010] argumentam que planejar e conduzir um processo de DP não é apenas "adicionar usuários ao processo", pois o potencial usuário precisa estar ativamente envolvido.

No que se refere ao público idoso, engajá-los ao processo é bastante desafiador [Lindsay et al. 2012], pois a maioria das técnicas e métodos existentes no DP não considera as barreiras cognitivas [Hendriks et al. 2013] e por isso, muitas vezes, precisam ser adaptadas para uso com/por pessoas idosas [Muriana and Hornung 2016].

Mais desafiador ainda é não só engajar pessoas em processos de DP, mas também torná-los parceiros de design, uma vez que, esse processo está relacionado à criatividade de designers e de não designers em colaboração. Nesse contexto, os não designers contribuem como "especialistas da própria experiência" [Sanders and Stappers 2008, p.12], colaborando em todas as etapas do processo, gerando um produto que se alinhe às suas necessidades.

Mas para engajá-los em todas as fases de um processo de codesign, partimos do pressuposto de que é necessário conhecer e reconhecer os participantes. Dessa forma, o significado da palavra "empatia", "a arte de se colocar no lugar do outro por meio da imaginação, compreendendo seus sentimentos e perspectivas e usando essa compreensão para guiar as próprias ações" [Krznaric 2015, p.10] se torna relevante e, quiçá, primordial para favorecer o engajamento do sujeito idoso em um processo de codesign de interação de tecnologias digitais.

Diante disso, escolhemos usar inicialmente a versão do SPIDe (SemioParticipatory Interaction Design Process) [Rosa and Matos 2016] adaptada por [Zabot et al. 2019]. Isso porque nessa versão a empatia foi incluída como elemento inicial do processo.

No entanto, em se tratando de sujeitos idosos, percebemos a necessidade de conceber uma nova estrutura do SPIDe, em que a empatia permeia todas as etapas. Para isso, foi realizado um estudo de caso, dentro de uma pesquisa aprovada pelo Comitê de Ética em Pesquisa da Universidade Estadual do Sudoeste da Bahia - UESB, sob o registro $\mathrm{n}^{\mathrm{o}}$ CAAE:17517019.2.0000.0055. 
Este artigo apresenta o relato dessa experiência com 10 mulheres idosas longevas (80+) engajadas em um processo de codesign de interação que possibilitou às participantes o despertar de novas habilidades e o interesse em estarem incluídas digitalmente.

O texto a seguir está estruturado da seguinte forma: a Seção 2 apresenta o processo de codesign utilizado: o SPIDe. A Seção 3 aborda sobre trabalhos relacionados. A Seção 4 descreve a metodologia utilizada. Na Seção 5 são apresentados os resultados. Na Seção 6 são discutidos alguns desafios e lições aprendidas. Por fim, na sexta e última seção, traçamos as considerações finais, seguidas das referências.

\section{SPIDe}

O SPIDe (Semio-Participatory Interaction Design Process) é um processo de Design de Interação Semioparticipativo que associa práticas participativas com a fundamentação teórica conceitual de Engenharia Semiótica. O papel principal do design de interação, conforme argumentam [Preece et al. 2015, p. 28], é "[...]criar experiências que melhorem e estendam a maneira como as pessoas trabalham, se comunicam e interagem".

Com base no Design Centrado na Comunicação (DCC) [Barbosa and Silva 2010], a versão inicial do SPIDe [Rosa and Matos 2016] foi composta de três etapas: análise de contexto, engenharia de interface e avaliação, conforme apresentado na Figura 1.

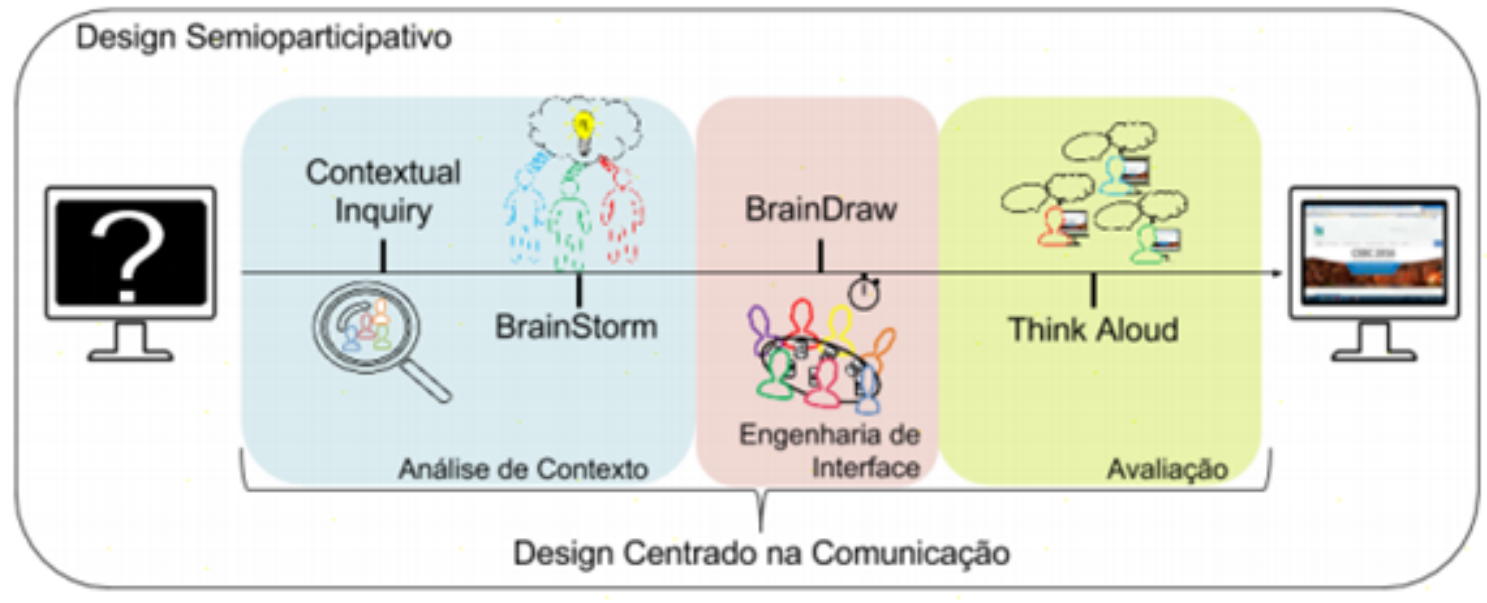

Figura 1. SPIDe (Rosa e Matos, 2016)

Utilizando o SPIDe com crianças surdas, foi necessário fazer uma adptação e assim [Zabot et al. 2019] adicionaram, antes da fase Análise de Contexto, a fase de Empatia, conforme apresentado na Figura 2.

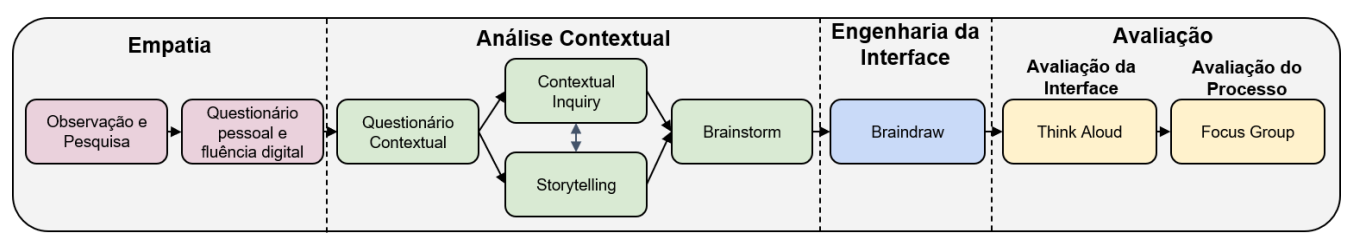

Figura 2. Versão adaptada do SPIDe por (Zabot, Andrade e Matos 2019) 


\section{Trabalhos Relacionados}

Alguns estudos que envolveram sujeitos idosos na concepção de um produto tiveram também a preocupação em reduzir as barreiras das tecnologias digitais, como é o caso do estudo de [Kopeć et al. 2018] que propuseram o método SPIRAL (Support for Participant Involvement in Rapid and Agile software development Labs). Nesse método, o sujeito idoso vai participando das diversas etapas do processo de desenvolvimento, à medida que as suas habilidades no uso de tecnologias digitais vão aumentando.

[Binda et al. 2018] ofereceram oficinas de compartilhamento de fotos e uso do Facebook(C), como estratégia para ajudar os idosos a se familiarizarem com a tecnologia, ao mesmo tempo, em que a equipe criava um "laço de confiança", antes de desenvolver atividades de design participativo. No entanto, os autores relatam que essa estratégia trouxe alguns desafios e dificuldades que eles tiveram em direcionar as conversas para gerar insights para a pesquisa de design.

[Martin-Hammond et al. 2018] incluíram uma sessão de crítica, em que os participantes criticaram um site interativo que fornece informações sobre saúde. Essa sessão visou apresentar aos participantes a ideia de avaliar o design de uma interface, considerando seus benefícios e problemas, como também usaram a sessão de crítica como um contato inicial para preparar os participantes para a atividade posterior de design.

Assim, como esses estudos, o presente trabalho concentrou também seus esforços em reduzir as barrerias das tecnologias, no entanto o que diferencia dos outros estudos é que nenhum deles ofereceu treinamento de tecnologias digitais integrado ao processo, visando a inclusão sociodigital, por meio de uma metodologia personalizada e direcionada às necessidades de cada participante.

\section{Metodologia}

Este estudo foi realizado no período de junho a dezembro de 2019 em uma turma de mulheres idosas residentes em uma instituição franciscana particular que abriga exclusivamente pessoas idosas do sexo feminino. As sessões de codesign ocorreram na própria instituição, em um local destinado às refeições diárias, todas as sextas-feiras, com a carga horária máxima de 2 horas diárias.

Participaram do estudo 10 mulheres idosas, em sua maioria longevas (80+) com idade mínima 82 anos e a máxima 97 anos. Havendo a participação também de duas mulheres idosas com 72 anos de idade. Todas as participantes faziam parte de um curso de uso de smartphone, oferecido pelo Grupo de Pesquisa e Extensão em Informática, Educação e Sociedade - Onda Digital, do qual os pesquisadores deste estudo fazem parte. Para a coleta de dados, utilizou-se: (i) uma entrevista individual mediada por um formulário $^{1}$; (ii) observação participante; (iii) conversas informais; e (iv) notas de campo com gravações de áudio.

As participantes tinham pouca ou nenhuma experiência com uso, tampouco desenvolvimento, de tecnologias digitais. Todas tinham aparelho telefônico móvel, sendo seis da categoria smartphone. Quanto à escolaridade, houve representação em três níveis:

\footnotetext{
${ }^{1}$ Roteiro de perguntas preenchido pelo entrevistador, no momento da entrevista, por meio do contato face a face entre pesquisador e informante.
} 
ensino fundamental I (duas participantes); ensino médio (seis participantes) e ensino superior (duas).

Com relação aos problemas de saúde, apesar dos problemas declarados pelas participantes, a exemplos de: diabete, artrite, artrose, início de Alzheimer, entre outros, nenhuma era incapaz funcionalmente ${ }^{2}$. Por isso, neste estudo, as participantes são consideradas mulheres idosas independentes. Para preservar a identidade das participantes, elas foram identificadas por um código (P1, P2...P(n)).

Também foram envolvidos neste estudo três pesquisadores (uma doutoranda em Ciência da Computação e dois estudantes de graduação em Sistemas de Informação), que se revezaram no papel de facilitadores; um mestre e uma mestranda em Ciência da Computação, no papel de monitores e sete graduandos, do curso de Bacharelado Interdisciplinar (BI) em Saúde no papel de monitores e observadores, que participaram em momentos distintos das etapas.

\subsection{Nova estrutura do SPIDe}

Para conduzir um estudo com pessoas idosas, foi utilizada inicialmente a versão do SPIDe adaptada por [Zabot et al. 2019]. No entanto, no decorrer do estudo, houve a necessidade de se fazer uma nova adaptação, em que a Empatia não é compreendida mais como uma etapa inicial, mas como um "fio invisível"que costura todo o trabalho intersubjetivo em campo, permeando todas as etapas do processo [Minayo and Costa 2019, p.22]. Além dessa alteração, foram adicionadas as etapas "Envolvimento" e "Preparação para o Design". A nova estrutura do SPIDe se diferencia das versões anteriores também por ser uma estrutura cíclica, iterativa e composta de seis etapas, conforme Figura 3.

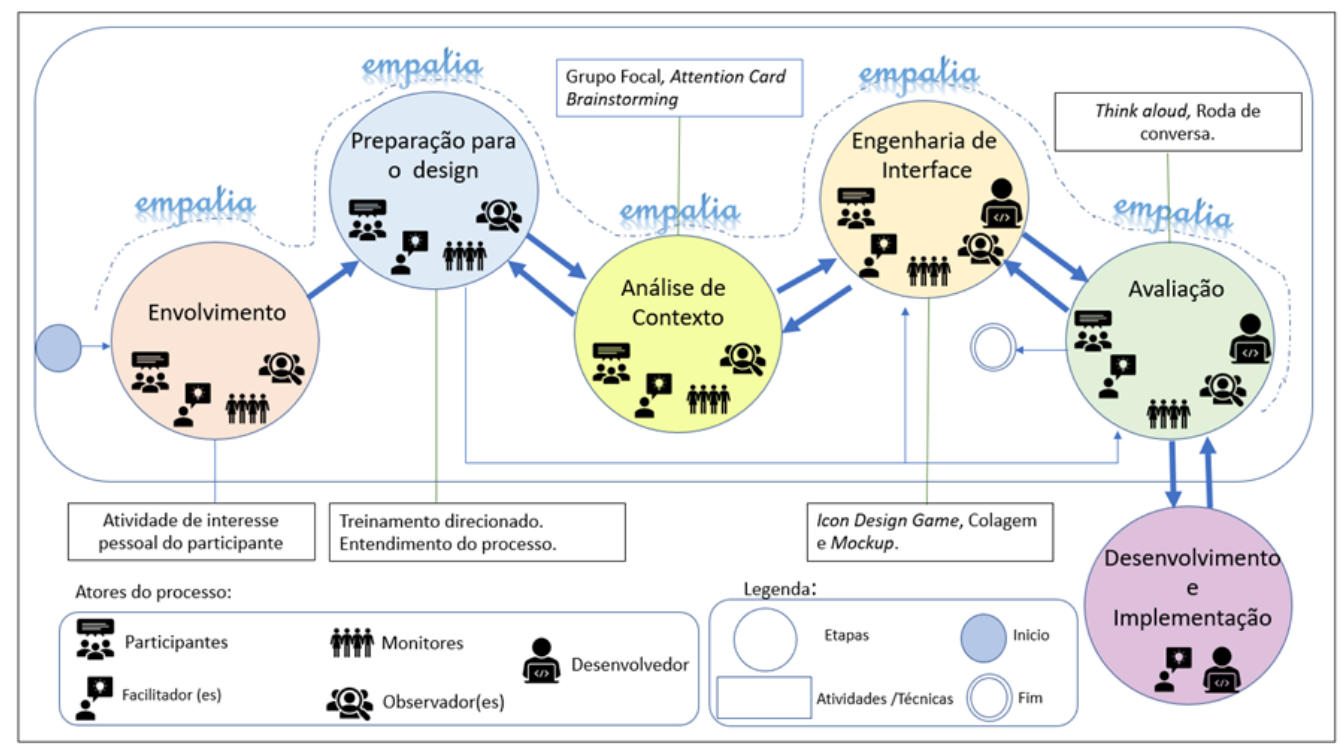

Figura 3. Nova estrutura do SPIDe

Para essa nova estrutura, além das participantes, foram necessários os seguintes atores: (i) facilitador - responsável em conduzir todo o processo de codesign de interação; (ii) observador - responsável em observar, fazer anotações escritas e realizar gravações de

\footnotetext{
${ }^{2}$ Ser dependente da ajuda de alguém para viver
} 
áudio/vídeo; (iii) monitores - responsáveis em auxiliar os participantes no desenvolvimento das atividades); (iv) desenvolvedor - responsável em desenvolver o produto ideado e prototipado pelas participantes. A seguir, uma breve descrição das etapas do processo.

Etapa 1: Envolvimento - visa entender as necessidades, expectativas, pensamentos e sentimentos de visão de mundo do sujeito idoso, estabelecendo uma relação empática, por meio de atividades de interesse pessoal do sujeito idoso. Neste estudo, a atividade de interesse das participantes foi relacionada ao treinamento no uso de smartphone/celular.

Etapa 2: Preparação para o design - essa etapa possibilita aos sujeitos idosos compreenderem o projeto, elementos de interface, a interação, preparando-os para as atividades de design de interação.

Etapa 3: Análise de Contexto - nesta etapa, por meio de identificação de problemas e/ou necessidades, os sujeitos idosos determinam o artefato a ser projetado e sugerem soluções com relação ao contexto de uso do artefato a ser projetado. Etapa 4: Engenharia de interface - nesta etapa são produzidos protótipos de design de interação pelos sujeitos idosos.

Etapa 5: Avaliação - o protótipo produzido anteriormente é avaliado pelos sujeitos idosos.

Etapa 6: Desenvolvimento e Implementação - etapa final, em que será desenvolvido/implementado o produto prototipado na etapa anterior. Após finalizado, o produto retornará à etapa de Avaliação para ser validado pelo grupo. Caso o produto apresente problemas durante a avaliação, esses problemas deverão ser anotados pelos desenvolvedores que farão a correção e irão encaminhar novamente para a avaliação, gerando um ciclo até o produto esteja funcional e maduro. Essa foi a única etapa em que as participantes não estiveram envolvidas.

Para alcançar os objetivos das etapas, várias sessões de codesign de interação foram realizadas e várias técnicas com abordagem participativa foram utilizadas. O número de participantes foi distinto, variando entre 4 a 8 mulheres idosas. A seguir, serão descritas algumas técnicas que promoveram o despertar das possibilidades e competências das participantes idosas ao desejarem ser incluídas digitalmente.

Grupo focal - é uma discussão moderada que normalmente envolve de 6 a 12 participantes [Gaskell 2002], com o intuito de explorar um conjunto específico de assuntos a partir de uma questão focal.

Attention Card - é uma técnica criada para visualizar situações/cenários da vida cotidiana dos idosos [Frennert et al. 2012].

Brainstorming - possibilita o envolvimento das pessoas em uma "tempestade de ideias", sugerindo novas e melhores ideias. Essa técnica foi sugerida na versão original do SPIDe [Rosa and Matos 2016].

Protótipo com colagem - "colagem, é uma técnica que apoia expressão criativa, mas não exige que os participantes tenham previamente competências artísticas ou técnicas" [McKay et al. 2006, p.1]. Por meio dessa técnica os participantes produzem protótipos de baixa fidelidade. 
Mockups - usados para obter feedback dos usuários sobre funcionalidades/usabilidade/compreensão da ideia básica de design [Folmer 2014].

\section{Resultados e Discussão}

Na etapa de "envolvimento", foram identificadas as principais dificuldades das participantes referentes ao uso de smartphone/celular e aplicativos. Nessa etapa, apesar de a maioria das participantes possuir smartphone, apenas uma delas tinha alguma experiência no uso de aplicativos (uso de redes sociais, email, internet bank, câmera fotográfica), as demais já haviam experienciado o WhatsApp(c) ou não tinham experiência alguma com uso de smartphones ou aplicativos. Essas participantes não tinham smartphone, mas celulares convencionais.

Na segunda etapa (preparação para o design), realizamos atividades em papel impresso, para as participantes entenderem os ícones, as telas e as formas de interação em um aplicativo. Para isso, imprimimos vários ícones e telas relacionadas ao WhatsApp(c).

Durante as duas primeiras etapas, foram realizados treinamentos para o uso de smartphone/celular, por meio de uma metodologia individualizada e direcionada a trabalhar com as necessidades que cada participante sinalizava como prioridade naquele momento e espaço de aulas. O planejamento de conteúdo era flexível, visando o aprendizado de uso do WhatsApp(c), transferência bancária, Youtube(C), fotos, fazer e receber ligações.

$\mathrm{Na}$ terceira etapa (análise de contexto), por meio das técnicas grupo focal, attention card e brainstorming, foram definidos tanto o artefato a ser ideado quanto o seu contexto de uso. O grupo focal oportunizou aos pesquisadores/facilitadores compreenderem o comportamento das participantes no falar, no sentir e no agir diante a um grupo. Isso influenciou a escolha e planejamento das próximas técnicas.

A técnica attention card possibilitou objetividade nas falas, gerando um artefato a ser ideado. Com a técnica de brainstorming, as ideias do contexto de uso do artefato definido foram surgindo e com elas várias experiências vivenciadas pelas participantes foram lembradas e compartilhadas, gerando nelas uma mistura de alegria e saudades.

$\mathrm{Na}$ quarta etapa (engenharia de interface), por meio das técnicas de prototipagem com colagem e mockups, constatamos a participação ativa das mulheres idosas ao produzirem seus próprios protótipos do aplicativo. Por meio da técnica de colagem, as participantes escolheram e colaram os ícones que representavam os signos que fazem parte dos seus sistemas de significação, para compor as telas do aplicativo, conforme exibido na Figura 4.

Com base nos protótipos criados pelas participantes, foram desenvolvidos mockups que continham elementos de interface de cada protótipo. Os mockups proporcionaram às participantes visualizarem, de modo mais próximo do real, o que elas tinham criado por meio da técnica de colagem. Isso gerou sentimentos de satisfação e empoderamento, principalmente nas participantes que não se interessavam em ter (ou aprender a usar) um smartphone, por se achar "[...] velha demais para aprender" (P1, 85 anos); (P2, 83 anos).

Produzir um protótipo e visualizar elementos escolhidos por elas, compondo as telas de um mockup, despertou o desejo de estarem incluídas digitalmente, pelo simples 


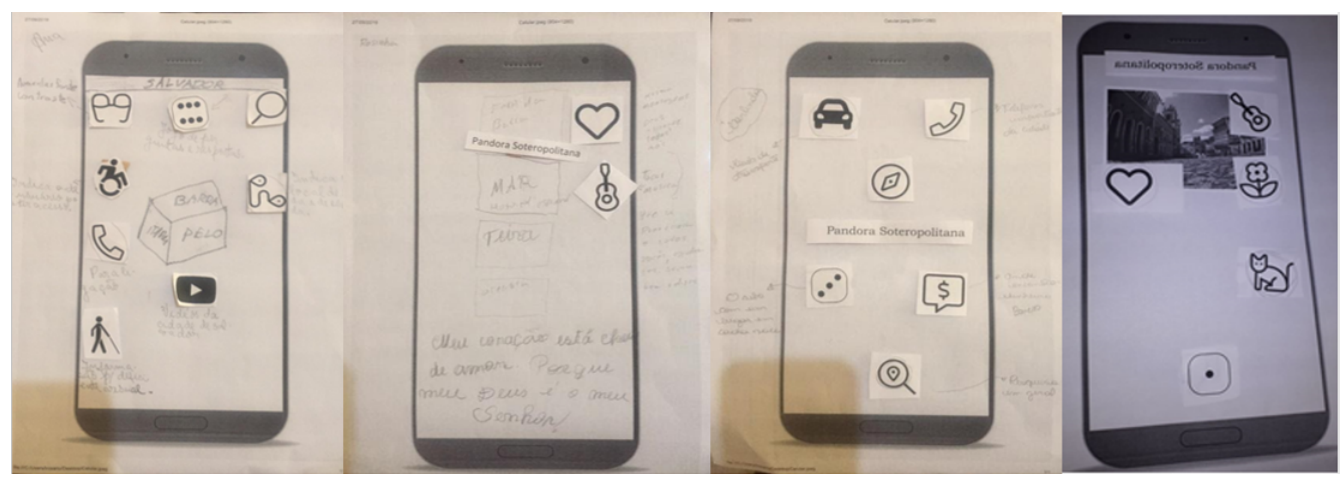

Figura 4. Exemplos dos Protótipos de papel da tela incial, desenvolvidos pelas participantes.

fato de compreenderem que são capazes de "criar" e aprender a usar um aplicativo, constatado em uma das falas de uma das participantes: "[...] eu tô achando excelente as três últimas aulas. Antes eu não entendia nada! Mas agora eu entendi tudo de um aplicativo! Agora sim, vou pedir para meu filho comprar um desse pra mim.” (P1, 85 anos).

Por fim, na etapa de avaliação, as participantes puderam avaliar o protótipo desenvolvido, interagindo com o aplicativo ideado e prototipado por elas. Nessa etapa, notamos o quanto os declínios cognitivos, sensoriais e motores, do processo natural de envelhecimento, dificultam a interação com a tecnologia digital.

As atividades desenvolvidas ao longo do processo, por meio de uma metodologia individualizada e direcionada às necessidades, associadas, às características empáticas dos pesquisadores e monitores favoreceram as participantes se sentirem mais seguras, confiantes e se expressarem de forma mais natural, sem restrições ou constrangimentos.

Isso possibilitou que elas percebessem o seu valor na atividade, os benefícios da tecnologia em si e da participação delas, retratados nas seguintes falas: "é bom para memória, incentiva[...]" (P3, 89 anos); "é melhor estar aqui do que presa no quarto" (P4, 85 anos); "precisamos estar com as tecnologias sob o nosso domínio" (P6, 72 anos).

\section{Desafios e Lições aprendidas}

Incluir digitalmente mulheres idosas, por meio de um processo de codesign de interação para produção de tecnologias digitais foi bastante desafiador. Dentre os desafios, destacamos inicialmente a importância de fazê-las compreender o quanto elas são capazes de aprender tecnologia, elevar a autoestima delas e a percepção do seu valor dentro da pesquisa e na sociedade.

Outro desafio foi com relação às escolhas de técnicas que pudessem gerar nelas sentimentos de bem-estar, empoderamento e pertencimento. O processo de codesign de interação utilizado foi configurado para que o engajamento das participantes ao processo e consequentemente a sua inclusão no mundo digital, ocorressem de forma genuína e positiva.

Aprendemos de uma forma geral, a importância do saber escutar com respeito e sem julgamentos, de conduzir um processo flexível e direcionado às necessidades e expectativas das participantes, disponibilizar um acompanhamento individual e personalizado, entre outras. 
IX Congresso Brasileiro de Informática na Educação (CBIE 2020)

Anais do XXVI Workshop de Informática na Escola (WIE 2020)

\section{Considerações finais}

Ao longo do processo de codesign de interação, observamos o quanto as participantes foram se empoderando e sentimentos iniciais de incapacidade por serem "velhas demais para aprender" foram sendo substituídos por sentimentos de alegria, de pertencimento, empoderamento e novas habilidades iam sendo experimentadas por elas, ao colaborarem com a criação de um aplicativo.

A relação empática estabelecida desde o início, e que foi se fortalecendo ao longo do processo, possibilitou escolhas de técnicas que promoveram participação mais efetiva nas atividades de design, contribuindo para autoestima e despertando mais ainda o interesse em estarem incluídas digitalmente.

Os próximos passos nos direcionam a refinar as atividades de inclusão digital realizadas ao longo do processo, visando contemplar uma maior apropriação da tecnologia. Espera-se com este trabalho contribuir com outras iniciativas e estudos no campo da inclusão sociodigital de mulheres idosas.

\section{Referências}

Barbosa, S. and Silva, B. (2010). Interação humano-computador. Elsevier Brasil.

Binda, J., Wang, X., and Carroll, J. M. (2018). Recruiting older adults in the wild: reflections on challenges and lessons learned from research experience. In Proceedings of the 12th EAI International Conference on Pervasive Computing Technologies for Healthcare, pages 290-293.

Bizelli, M. H. S., Barrozo, S., Tanaka, J. S., and Sandron, D. C. (2009). Informática para a terceira idade-características de um curso bem sucedido. Revista Ciência em Extensão, 5(2):4-14.

Folmer, E. (2014). The glossary of human computer interaction:mockups. Interaction Design Foundation.

Frennert, S., Östlund, B., and Eftring, H. (2012). Capturing seniors' requirements for assistive robots by the use of attention cards. In Proceedings of the 7th Nordic Conference on Human-Computer Interaction: Making Sense Through Design, pages 783-784.

Gaskell, G. (2002). Entrevistas individuais e grupais. Pesquisa qualitativa com texto, imagem e som: um manual prático, 2:64-89.

Hendriks, N., Truyen, F., and Duval, E. (2013). Designing with dementia: Guidelines for participatory design together with persons with dementia. In IFIP Conference on Human-Computer Interaction, pages 649-666. Springer.

IBGE (2018). número de habitantes do país deve parar de crescer em 2047. Editora: Estatísticas Sociais.

Kopeć, W., Nielek, R., and Wierzbicki, A. (2018). Guidelines towards better participation of older adults in software development processes using a new spiral method and participatory approach. In Proceedings of the 11th International Workshop on Cooperative and Human Aspects of Software Engineering, pages 49-56.

Krznaric, R. (2015). O poder da empatia: a arte de se colocar no lugar do outro para transformar o mundo. Editora Schwarcz-Companhia das Letras. 
Lindsay, S., Brittain, K., Jackson, D., Ladha, C., Ladha, K., and Olivier, P. (2012). Empathy, participatory design and people with dementia. In Proceedings of the SIGCHI Conference on Human Factors in Computing Systems, pages 521-530.

Martin-Hammond, A., Vemireddy, S., and Rao, K. (2018). Engaging older adults in the participatory design of intelligent health search tools. In Proceedings of the 12th EAI International Conference on Pervasive Computing Technologies for Healthcare, pages 280-284.

McKay, D., Cunningham, S. J., and Thomson, K. (2006). Exploring the user experience through collage. In Proceedings of the 7th ACM SIGCHI New Zealand chapter's international conference on Computer-human interaction: design centered HCI, pages 109-115.

Minayo, M. C. d. S. and Costa, A. P. (2019). Técnicas que fazem o uso da palavra, do olhar e da empatia: Pesquisa qualitativa em ação. In Técnicas que fazem o uso da palavra, do olhar e da empatia: Pesquisa Qualitativa em Ação, pages 63-63.

Mol, A. M. (2011). Recomendações de usabilidade para interface de aplicativos para smartphones com foco na terceira idade. Pontifícia Universidade Católica de Minas Gerais-Belo Horizonte.

Muller, M. J. and Druin, A. (2010). Participatory design: the third space in hci. humancomputer interaction: Development process. j. jacko and a. Sears. Eds. Handbook of HCI.

Muriana, L. M. and Hornung, H. (2016). Who are you? getting to know and understanding older adults with dementia in participatory design at a nursing home. In Proceedings of the 15th Brazilian Symposium on Human Factors in Computing Systems, pages $1-10$.

Organization, W. H. et al. (2005). Envelhecimento ativo: uma política de saúde.

Pasqualotti, P. R., Pérez, C. C. C., Bez, M. R., and Klein, C. (2007). Inclusão digital para terceira idade: Oportunidades, possibilidades e propostas inovadoras. Technology, pages $1-11$.

Preece, J., Sharp, H., and Rogers, Y. (2015). Interaction design: beyond human-computer interaction. John Wiley \& Sons.

Rosa, J. C. and Matos, E. (2016). Semio-participatory framework for interaction design of educational software. In Proceedings of the 15th Brazilian Symposium on Human Factors in Computing Systems, pages 1-10.

Sanders, E. B.-N. and Stappers, P. J. (2008). Co-creation and the new landscapes of design. Co-design, 4(1):5-18.

Simonsen, J. and Robertson, T. (2012). Routledge international handbook of participatory design. Routledge.

Zabot, D., Andrade, S., and Matos, E. (2019). Game design participativo com crianças surdas e com deficiência auditiva: uma experiência no ensino fundamental. In Anais do I Workshop sobre Interação e Pesquisa de Usuários no Desenvolvimento de Jogos, pages 49-58. SBC. 